\title{
A novel dynamic model for predicting outcome in patients with hepatitis $B$ virus related acute-on-chronic liver failure
}

\author{
Ran Xue ${ }^{1, *}$, Zhonghui Duan ${ }^{1, *}$, Haixia Liu ${ }^{1}$, Li Chen ${ }^{2}$, Hongwei $\mathbf{Y u}^{1}$, Meixin Ren ${ }^{1}$, \\ Yueke Zhu ${ }^{1}$, Chenggang Jin ${ }^{3}$, Tao Han ${ }^{4}$, Zhiliang Gao ${ }^{5}$ and Qinghua Meng ${ }^{1}$ \\ ${ }^{1}$ Department of Critical Care Medicine of Liver Disease, Beijing You-An Hospital, Capital Medical University, Beijing, China \\ ${ }^{2}$ Georgia Prevention Institute, Medical College of Georgia, Augusta University, Augusta, GA, USA \\ ${ }^{3}$ The School of Social Development and Public Policy, Beijing Normal University, Beijing, China \\ ${ }^{4}$ Department of Hepatology, Tianjin Third Central Hospital of Tianjin Medical University, Tianjin, China \\ ${ }^{5}$ Department of Infectious Diseases, The Third Affiliated Hospital, Zhongshan University, Guangzhou, China \\ *These authors have contributed equally to this work
}

Correspondence to: Qinghua Meng, email: meng_qh@126.com

Keywords: acute-on-chronic liver failure; hepatitis B; prognostic model

Received: October 06, $2017 \quad$ Accepted: October 27, $2017 \quad$ Published: November 14, 2017

Copyright: Xue et al. This is an open-access article distributed under the terms of the Creative Commons Attribution License 3.0 (CC BY 3.0), which permits unrestricted use, distribution, and reproduction in any medium, provided the original author and source are credited.

\section{ABSTRACT}

Aim: It is challenging to predict the outcome of patients with hepatitis B virus related acute-on-chronic liver failure (HBV-ACLF) through existing prognostic models. Our aim was to establish a novel dynamic model to improve the predictive efficiency of 30-day mortality in HBV-ACLF patients.

Methods: 305 patients who were diagnosed as HBV-ACLF (derivation cohort, $n=211$; validation cohort, $n=94$ ) were included in this study. The HBV-ACLF dynamic (HBV-ACLFD) model was constructed based on the daily levels of predictive variables in 7 days after diagnosis combined with baseline risk factors by multivariate logistic regression analysis. The HBV-ACLFD model was compared with the Child-TurcottePugh (CTP) score, end-stage liver disease (MELD) score, and MELD within corporation of serum sodium (MELD-Na) score by the area under the receiver-operating characteristic curves (AUROC).

Results: The HBV-ACLFD model demonstrated excellent discrimination with AUROC of 0.848 in the derivation cohort and of 0.813 in the validation cohort $(p=0.620)$. The performance of the HBV-ACLFD model appeared to be superior to MELD score, MELD-Na score and CTP score $(P<0.0001)$.

Conclusion: The HBV-ACLFD model can accurately predict 30-day mortality in patients with HBV-ACLF, which is helpful to select appropriate clinical procedures, so as to relieve the social and economic burden.

\section{INTRODUCTION}

Acute-on-chronic liver failure (ACLF) is a life-threatening syndrome with varied etiology and manifestations with a short-term mortality of $50-90 \%$ [1]. ACLF is defined as the acute decompensation of liver function in patients with either previously diagnosed or undiagnosed chronic liver disease [2-3]. Hepatitis B virus (HBV) is the leading cause of chronic liver disease in the Asia-Pacific region, including China and India [4]. Liver transplantation (LT) is a feasible and beneficial treatment for patients with ACLF to achieve survival [5]. It is important to accurately distinguish the ACLF patients who need LT, and grasp the best opportunity for LT [6]. To guide and optimize therapeutic strategy for ACLF patients, an accurate prognostic scoring system is prerequisite [7]. 
Varied prognostic scoring systems have been developed to predict ACLF mortality and guide the decision-making of LT. The Child-Pugh-Turcotte (CPT) classification and the model of end-stage liver disease (MELD) score are the most commonly used for patient priority on the waiting list of LT [8-9]. MELD Serum sodium (MELD-Na) score, as a modified MELD score, presented promising value for predicting mortality among patients on the LT waiting list [10-11].

However, each of the models above cannot apply reasonably to evaluate the clinical outcome in hepatitis $B$ virus related acute-on-chronic liver failure (HBVACLF), mainly due to differences in patient background queues. There exist great differences between Eastern and Western ACLF in definition and diagnostic criteria, involving the basis of chronic liver disease, the concept of organ failure and the diagnostic criteria for organ failure [12-13]. Furthermore, HBV-ACLF is a dynamic process in which the variables at the time of hospitalization are predicted to vary over time, accompanied with the clinical processes and outcomes change accordingly. The existing prognostic models were established based on static baseline, which cannot logically evaluate the predictive outcome [14]. Therefore, it is urgently needed to derivation and validation of a novel dynamic model for predicting outcome in patients with HBV-ACLF.

Our aim was to establish a prognostic model according to early changes of independent predictive variables at admission in patients with HBV-ACLF; to identify whether the model was superior to the existing prognostic models such as CPT scores, MELD scores, and MELD-Na scores; and ultimately to validate the model by a cohort of 94 patients with HBV-ACLF from three different geographical spread medical centers, so as to confirm the potential value of model for clinical treatment decision making.

\section{RESULTS}

\section{Baseline characteristics of patients}

305 patients who were diagnosed as HBVACLF (derivation cohort, $\mathrm{n}=211$; validation cohort, $n=94$ ) were included in this study. The comparisons of patients' characteristics in the derivation and validation cohorts were shown in Table 1. There was no significant difference in gender distribution, died numbers within 30-day, HE, ascites and laboratory parameters at baseline between derivation cohort and validation cohort. The preexisting chronic liver disease $(\mathrm{p}<0.001)$, infection $(\mathrm{p}<$ $0.001)$ and PT $(p=0.007)$ were significantly less in the validation cohort than those in the derivation cohort. The age $(p=0.016)$ and suspicion of infection $(p=0.005)$ were higher in the validation cohort than those in the derivation cohort.

\section{Predictors of mortality in the derivation cohort}

30-day mortality was applied as the end-point in the multivariate logistic regression analysis, and all variables in univariate analyses were imported into the model. In this model, TBiL, albumin, HE, INR, blood neutrophils percentage count, and suspicion of infection were independent risk factors for death. The $\beta$-coefficient, OR and $95 \%$ CI for independent predictors were presented in Table 2.

\section{The development of prognostic model}

The prognostic model derived from the derivation cohort (Table 2). The initial model was established based on the independent predictors of admission mortality. The model passed the Hosmer-Lemeshow goodness-offit test ( $\mathrm{p}=0.535)$; the area under the Receiver-operating characteristic (AUROC) curve was also used. However, its discriminative ability was only modest (AUROC 0.745 , $95 \%$ CI: 0.667 to 0.823 ). Ultimately, the HBVACLF dynamic (HBV-ACLFD) model was constructed based on the independent predictors of mortality and the daily changes within 7 days after diagnosis (Table 3 ). The Hosmer-Lemeshow goodness-of-fit test for HBV-ACLFD model was also performed $(\mathrm{p}=0.288)$. The HBV-ACLFD model was discriminated (AUROC $=0.848,95 \%$ CI: 0.793 to 0.902 ; Figure $1 \mathrm{~A}$ ). The specificity, sensitivity, negative predictive value and positive predictive value of the HBV-ACLFD model were $93.63 \%, 44.44 \%, 83.05 \%$ and $70.59 \%$, respectively.

\section{The validation of the predicted model}

The HBV-ACLFD model retained a good discrimination when applied to a validation cohort (AUROC $=0.813 ; 95 \%$ CI, 0.720 to 0.905 ; Figure 1B). The Hosmer-Lemeshow goodness-of-fit test for HBV-ACLFD model was also performed $(\mathrm{p}=0.612)$. The AUROC from three different medical centers were provided, respectively (Figure 2).

\section{Comparison with alternative predicted models}

By the 30-day mortality and c-statistic as the endpoint, we identified four post-admission prognostic models of AUROC. The overall 30-day mortality was $25.59 \%$ ( $\mathrm{n}=54$ of 211 ) (Supplementary Figure 1). On admission, the AUROC was highest for the HBV-ACLFD model (0.848), followed by the MELD ( $0.696 ; 95 \%$ CI, 0.609 to 0.784$)$, MELD-Na $(0.686 ; 95 \% \mathrm{CI}, 0.597$ to $0.776)$, and CTP $(0.566 ; 95 \%$ CI, 0.471 to 0.660$)$ scores (Figure 3). There were significant differences between all pairs of scores for the dynamic changes $(p<0.05)$. 
Table 1: Clinical profiles of patients with HBV-ACLF in the derivation and validation cohorts

\begin{tabular}{|c|c|c|c|c|}
\hline Parameters & & $\begin{array}{l}\text { Derivation cohort } \\
(n=211)\end{array}$ & $\begin{array}{l}\text { Validation cohort } \\
(n=94)\end{array}$ & P values \\
\hline \multirow{4}{*}{ Character } & Age (years), mean \pm SD & $43.94 \pm 12.63$ & $47.15 \pm 10.60$ & $0.016^{*}$ \\
\hline & Male, n $(\%)$ & $176(83.41)$ & $81(86.17)$ & 0.541 \\
\hline & Died within 30 days, n (\%) & $54(25.59)$ & $33(35.11)$ & 0.089 \\
\hline & None HE, n (\%) & $152(72.04)$ & $61(64.89)$ & \\
\hline \multirow[t]{2}{*}{$\mathrm{HE}^{\mathrm{a}}$} & I-II HE, n (\%) & $41(19.43)$ & $24(25.53)$ & 0.429 \\
\hline & III-IV HE, n (\%) & $18(8.53)$ & $9(9.57)$ & \\
\hline \multirow{2}{*}{$\begin{array}{l}\text { Pre-existing chronic liver } \\
\text { disease }^{\text {b }}\end{array}$} & Chronic hepatitis B, n (\%) & $167(79.15)$ & $49(52.13)$ & \multirow{2}{*}{$<0.001^{*}$} \\
\hline & Compensated liver cirrhosis, n (\%) & $44(20.85)$ & $45(47.87)$ & \\
\hline \multirow{6}{*}{ In-hospital complication } & \multirow{5}{*}{$\begin{array}{l}\text { None, n (\%) } \\
\text { Mild, n (\%) } \\
\text { Moderate, n (\%) } \\
\text { Severe, n }(\%)\end{array}$} & $67(31.75)$ & $26(27.66)$ & \multirow{4}{*}{0.184} \\
\hline & & $78(36.97)$ & $27(28.72)$ & \\
\hline & & $47(22.27)$ & $27(28.72)$ & \\
\hline & & $19(9.00)$ & $14(14.89)$ & \\
\hline & & $12(5.68)$ & $28(29.78)$ & $<0.001^{*}$ \\
\hline & Suspicion of infection, $\mathrm{n}(\%)^{\mathrm{c}}$ & $77(36.49)$ & $19(20.21)$ & $0.005^{*}$ \\
\hline \multirow{11}{*}{$\begin{array}{l}\text { Laboratory parameters at } \\
\text { baseline }\end{array}$} & ALT (U/L), median (range) & $\begin{array}{c}163.50 \\
(13.70-2550.00)\end{array}$ & $\begin{array}{c}197.75 \\
(19.00-7822.60)\end{array}$ & 0.227 \\
\hline & AST (U/L), median (range) & $\begin{array}{c}161.35 \\
(31.50-5715.00)\end{array}$ & $\begin{array}{c}188.35 \\
(21.00-5688.20)\end{array}$ & 0.118 \\
\hline & TBil $(\mu \mathrm{mol} / \mathrm{L})$, median (range) & $\begin{array}{c}347.90 \\
(171.93-966.40)\end{array}$ & $\begin{array}{c}325.75 \\
(172.50-780.30)\end{array}$ & 0.446 \\
\hline & INR, median (range) & $\begin{array}{c}2.55 \\
(1.08-5.04)\end{array}$ & $\begin{array}{c}2.37 \\
(1.32-9.00)\end{array}$ & 0.127 \\
\hline & $\mathrm{Na}(\mathrm{mmol} / \mathrm{L})$, median (range) & $\begin{array}{c}135.00 \\
(111.00-145.40)\end{array}$ & $\begin{array}{c}135.00 \\
(113.50-143.70)\end{array}$ & 0.840 \\
\hline & $\operatorname{Albumin}(\mathrm{g} / \mathrm{L}), \operatorname{mean} \pm \mathrm{SD}$ & $31.00 \pm 4.73$ & $31.25 \pm 4.51$ & 0.667 \\
\hline & WBC $\left(\times 10^{9} / \mathrm{L}\right)$, median (range) & $\begin{array}{c}7.01 \\
(1.10-29.41)\end{array}$ & $\begin{array}{c}7.32 \\
(1.11-35.64)\end{array}$ & 0.489 \\
\hline & $\begin{array}{l}\text { Blood neutrophils percentage count, } \\
\text { median (range) }\end{array}$ & $\begin{array}{c}72.90 \\
(26.60-94.00)\end{array}$ & $\begin{array}{c}70.90 \\
(46.90-92.50)\end{array}$ & 0.438 \\
\hline & Hemoglobin $(\mathrm{g} / \mathrm{L})$, mean \pm SD & $119.63 \pm 23.74$ & $121.04 \pm 25.99$ & 0.642 \\
\hline & PLT $\left(\times 10^{9} / \mathrm{L}\right)$, median (range) & $\begin{array}{c}87.00 \\
(9.00-298.00)\end{array}$ & $\begin{array}{c}96.50 \\
(26.00-297.00)\end{array}$ & 0.077 \\
\hline & PTA, median (range) & $\begin{array}{c}29.50 \\
(8.00-39.50)\end{array}$ & $\begin{array}{c}32.00 \\
(9.00-40.00)\end{array}$ & 0.081 \\
\hline
\end{tabular}

\footnotetext{
a Mild to moderate hepatic encephalopathy (HE) was defined as grade I or II HE, severe HE was defined as grade III or IV HE, according to the West Haven classification.

$\mathrm{b}$ The pre-existing chronic liver disease patients were divided into two groups, counter balanced between liver cirrhosis and chronic hepatitis B.

${ }^{c}$ Suspicion of infection based on at least one of the following: WBC count $>10,000 / \mathrm{mm}^{3}$ or $\geq 50 \%$ increase with respect to baseline with a final value $>8,000 / \mathrm{mm}^{3}$; more than $5 \%$ of band forms; and/or temperature $>37.5^{\circ} \mathrm{C}$.

${ }^{*} \mathrm{P}<0.05$.
} 
Table 2: The initial model was based on independent predictors of mortality at admission.

\begin{tabular}{|c|c|c|c|c|c|}
\hline Variables & OR & CI1 & CI2 & $\beta$-coefficient & P values \\
\hline $\mathrm{Ln}(\mathrm{TBiL}, \mu \mathrm{mol} / \mathrm{L})$ & 3.07 & 1.23 & 7.65 & 1.12 & $0.016^{*}$ \\
\hline Ln (Albumin, g/L) & 0.98 & 0.91 & 1.06 & -0.02 & 0.667 \\
\hline Ln (INR) & 1.55 & 0.45 & 5.32 & 0.44 & 0.487 \\
\hline $\begin{array}{l}\text { Ln (Blood neutrophils } \\
\text { percentage count) }\end{array}$ & 1.04 & 1.01 & 1.08 & 0.04 & $0.012^{*}$ \\
\hline \multicolumn{6}{|l|}{$\mathrm{HE}$} \\
\hline I-II & 1.16 & 0.49 & 2.75 & 0.15 & 0.735 \\
\hline III-IV & 5.29 & 1.73 & 16.19 & 1.66 & $0.004^{*}$ \\
\hline Suspicion of infection ${ }^{\mathrm{a}}$ & 0.30 & 0.14 & 0.68 & -1.19 & $0.004^{*}$ \\
\hline Constant & 0.00 & 0.00 & 0.01 & -10.33 & $<0.001^{*}$ \\
\hline
\end{tabular}

${ }^{a}$ Suspicion of infection based on at least one of the following: WBC count $>10,000 / \mathrm{mm}^{3}$ or $\geq 50 \%$ increase with respect to baseline with a final value $>8,000 / \mathrm{mm}^{3}$; more than $5 \%$ of band forms; and/or temperature $>37.5^{\circ} \mathrm{C} .{ }^{*} \mathrm{P}<0.05$.

Table 3: The HBV-ACLFD model development based on predictors of mortality at baseline and their daily changes $(\Delta)$ within 7 days after diagnosis

\begin{tabular}{lccccc}
\hline Variables & OR & CI1 & CI2 & B-coefficient & P values \\
\hline Ln (TBiL, $\mu$ mol/L) & 3.02 & 0.99 & 9.22 & 1.11 & 0.052 \\
$\Delta$ Ln (TBiL, $\mu \mathrm{mol} / \mathrm{L})$ & 3928.33 & 1.71 & $9.02 \mathrm{e}+06$ & 8.28 & $0.036^{*}$ \\
Ln (Albumin, g/L) & 0.93 & 0.84 & 1.02 & -0.08 & 0.110 \\
$\Delta$ Ln (Albumin, g/L) & 0.69 & 0.46 & 1.02 & -0.38 & 0.063 \\
Ln (INR) & 2.25 & 0.56 & 9.05 & 0.81 & 0.255 \\
$\Delta$ Ln (INR) & 9910.49 & 23.74 & $4.14 \mathrm{e}+06$ & 9.20 & $0.003^{*}$ \\
Ln (Blood neutrophils & 1.06 & 1.02 & 1.10 & 0.06 & $0.001^{*}$ \\
percentage count) & & & & \\
$\Delta$ Ln (Blood neutrophils & 1.38 & 1.13 & 1.68 & 0.32 & $0.002^{*}$ \\
percentage count) & & & & \\
HE & 0.97 & 0.38 & 2.52 & -0.03 & 0.957 \\
$\quad$ I-II & 3.47 & 1.00 & 12.09 & 1.24 & 0.051 \\
$\quad$ III-IV & 0.37 & 0.15 & 0.87 & -1.00 & $0.022^{*}$ \\
Suspicion of infection & 0.00 & 0.00 & 0.02 & -10.27 & $0.002^{*}$ \\
Constant & & & & \\
\hline
\end{tabular}

${ }^{*} \mathrm{p}<0.05$.

\section{DISCUSSION}

HBV-ACLF can cause irreversible liver failure, leading to severe liver function damage. If LT is not available, it may lead to an at least $70 \%$ mortality rate [15]. Therefore, it is urgent to guide and optimize therapeutic strategy for HBV-ACLF patients. An accurate prognostic scoring system is a precondition for guiding and optimizing therapeutic strategy for HBV-ACLF patients. The results of our research showed that the HBV-ACLFD model exhibited excellent discrimination and almost the same performance in two cohorts (AUROC 0.848 in the derivation cohort and 0.813 in the validation cohort). The performance of the HBV-ACLFD model appeared to be 
superior to MELD score, MELD-Na score and CTP score $(\mathrm{P}<0.0001)$. It is indicated that the HBV-ACLFD model can accurately predict 30-day mortality in patients with HBV-ACLF, which is helpful to select appropriate clinical procedures for HBV-ACLF patients, so as to relieve the social and economic burden.

A number of definitions of ACLF have been put forward, based on advice from experts rather than evidence-based data. The definitions of heterogeneity show the differences from the etiology of liver disease in Eastern and Western countries [12]. In China, chronic HBV infection contributes to the ACLF. The early diagnosis and prompt treatment play a core role in the liver failure therapeutic strategies. In the western countries, ACLF is defined on the basis of compensated or decompensated cirrhosis [3]. Acute liver function decompensation mainly caused by alcohol abuse and bacterial infection. The therapeutic strategies of western countries focus on the multiple organ dysfunction syndrome (MODS), to distinguish the patients with

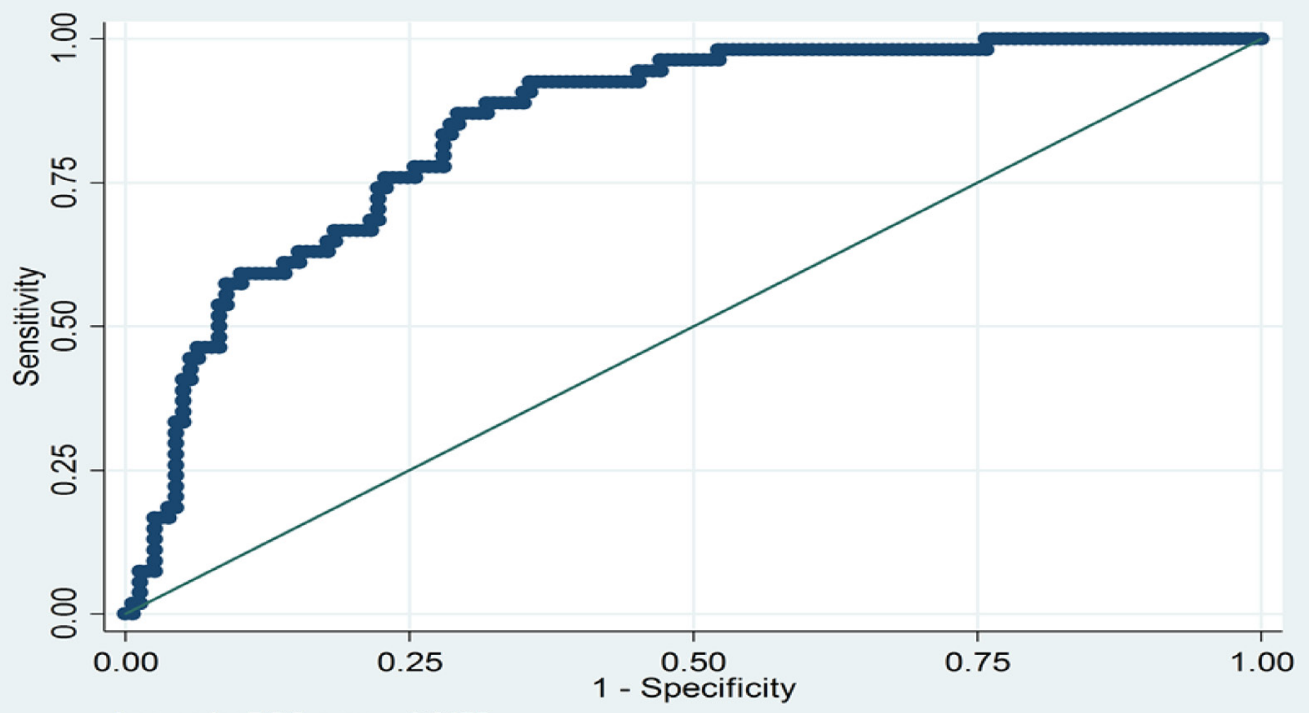

Area under ROC curve $=0.8475$

1 - Specificity

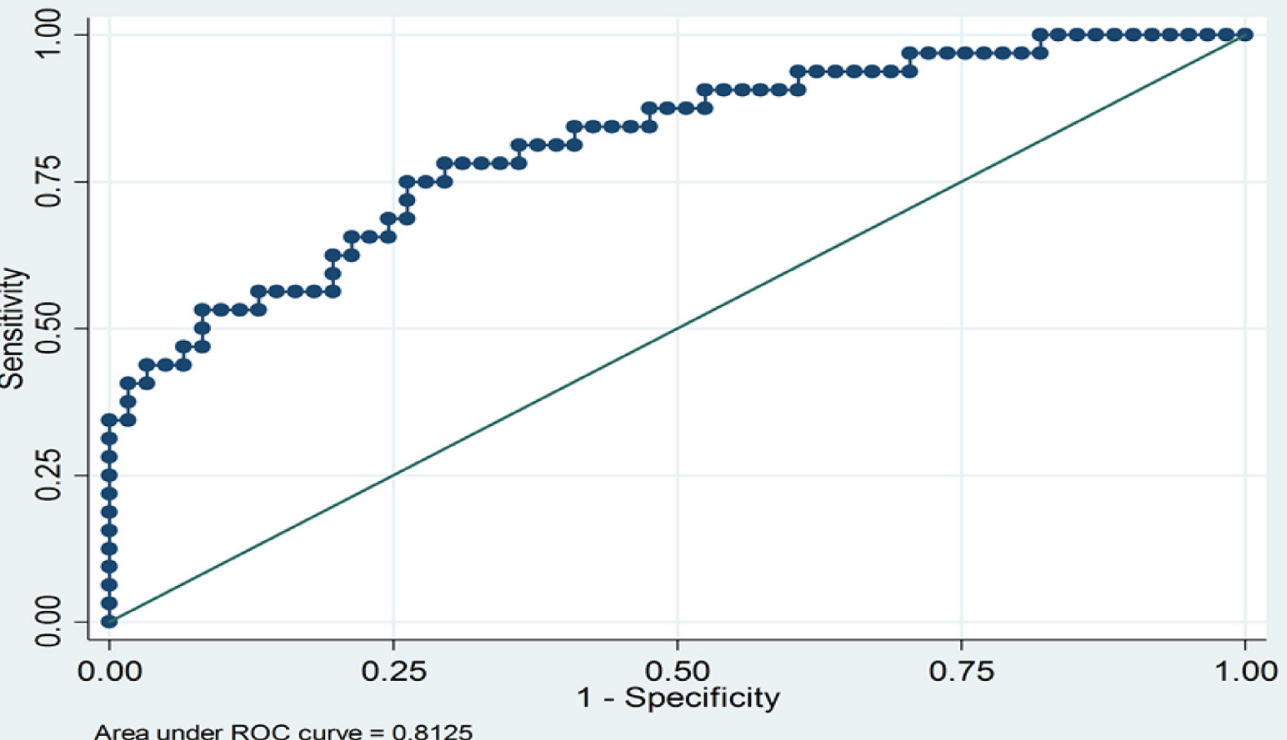

A

B

Figure 1: (A) Receiver operating characteristic curves of the HBV-ACLFD model in predicting mortality in the derivation cohort. The HBV-ACLFD model had discrimination (AUROC $=0.848$; 95\% CI: 0.793 to 0.902). (B) Receiver operating characteristic curves of the HBV-ACLFD model in predicting mortality in the validation cohort. The HBV-ACLFD model retained a good discrimination when applied to a validation cohort (AUROC $=0.813 ; 95 \%$ CI: 0.720 to 0.905 ). The Hosmer-Lemeshow goodness-of-fit test for HBV-ACLFD model has been performed $(p=0.612)$. 
high mortality risk who need to be treated in the ICU or moved to the waiting list of LT [16]. In addition, there are many differences of pathophysiology, clinical manifestation and prognosis between HBV related liver disease and alcoholic liver disease. Actually, in the AsiaPacific region, including China and India, ACLF is mainly induced by the HBV infection. Antiviral therapy is also the special treatment strategy in the comprehensive treatment of HBV related ACLF [12]. Therefore, it is necessary to build up an accurate prognostic scoring system based on HBV-ACLF patients specifically.

This study focuses on patients with HBV-ACLF from China. HBV-ACLF is one of the most lethal, costly, and pervasive diseases in China. Early predictors are essentially required to distinguish patients with ACLF who need orthotropic LT from those that can survive by only intensive medical care [17].

Currently, it remains difficult to identify appropriate indicators of poor outcome in HBV-ACLF [18]. The established prognostic models are mostly based on static baseline variables. However, patients' responses to the treatment could also affect the outcomes [19]. Thus, we retrospectively reviewed patients diagnosed as HBVACLF. Patients with HBV-ACLF were divided into a derivation cohort and a validation cohort. The data of patients with HBV-ACLF, including demographics, clinical, laboratory variables, underlying chronic liver disease, complications during the hospital course, as well as in-hospital special treatment, were collected. The derivation cohort was used to identify predictors of 30day mortality and construct the HBV-ACLFD prognostic model. $\Delta$ biomarker within 7 days after diagnosis was calculated and constructed into the model together with baseline risk factors based on logistic regression. The mortality rates in validation cohorts and derivation cohort with HBV-ACLF at 30-day after diagnosis were 25.59\% and $35.11 \%$, respectively, which are consistent with the results reported by Xia et al [20].

$\mathrm{HBV}-\mathrm{ACLF}$ is a dynamic process in which the variables at the time of hospitalization are predicted to vary over time, accompanied with the clinical processes and outcomes change accordingly. Meanwhile, prognosis predictions fluctuate over different clinical treatment. A study of acute liver failure (ALF) has shown that the model based on the early change of dynamic variables is better for the prediction than the model based on static baseline variables [21]. The high absolute values of AFP cannot predict the prognosis well, but the uptrend of AFP

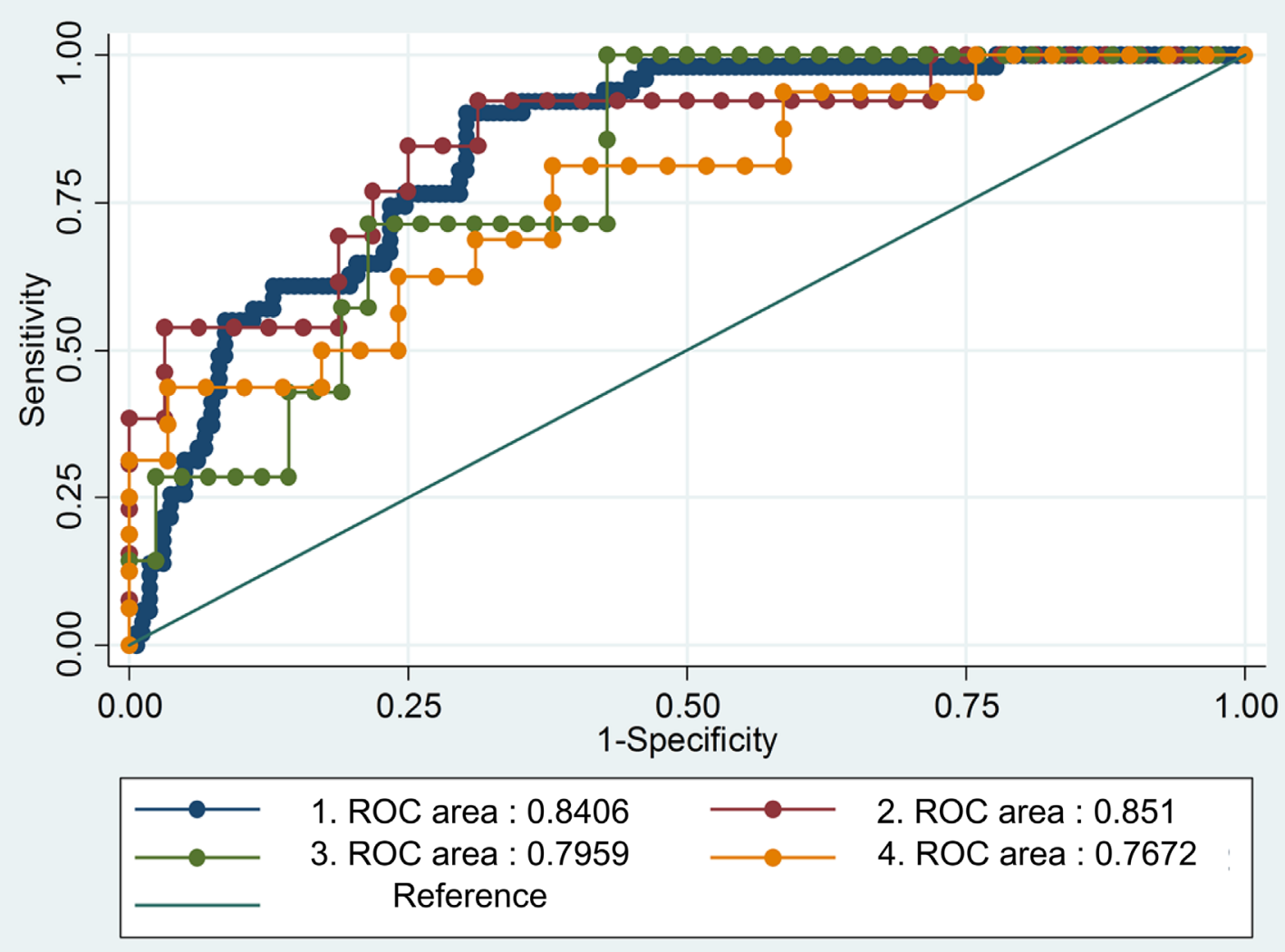

Figure 2: The AUROC from the three different medical centers. 1: Beijing You-an Hospital (derivation cohort), 2: the Third Affiliated Hospital of Sun Yat-Sen University, 3: Tianjin Third Central Hospital, 4: Beijing You-an Hospital (validation cohort). 


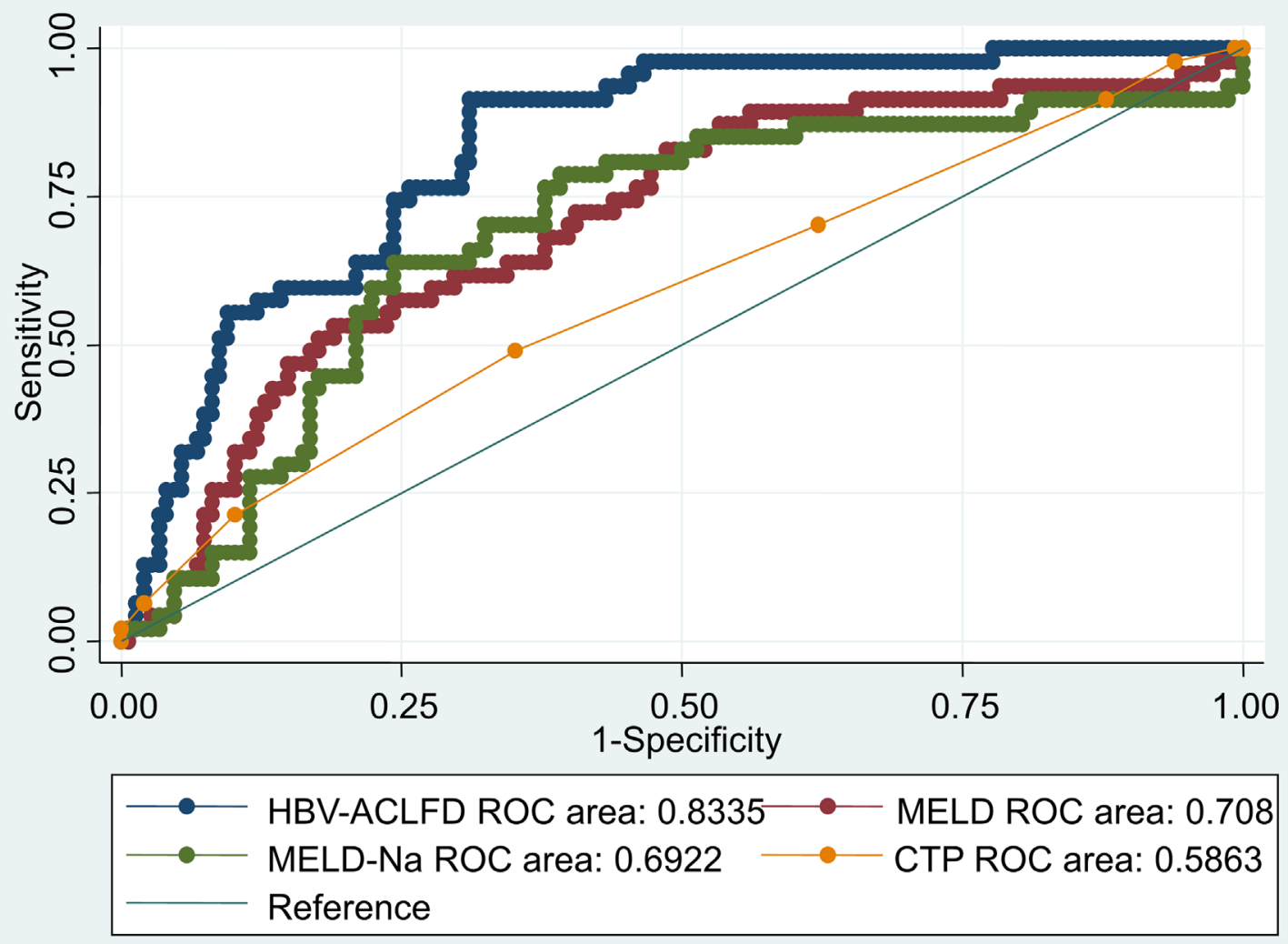

Figure 3: Comparison of the predictive accuracy for 30-day mortality between MELD, MELD-Na, CTP, and the dynamic prognostic model. On admission, the AUROC was highest for the HBV-ACLFD model (0.848), followed by the MELD (0.696; $95 \%$ CI, 0.609 to 0.784$)$, MELD-Na (0.686; $95 \%$ CI, 0.597 to 0.776$)$, and CTP (0.566; $95 \%$ CI, 0.471 to 0.660$)$ scores. There were significant differences between all pair of scores for the dynamic changes $(\mathrm{p}<0.05)$.

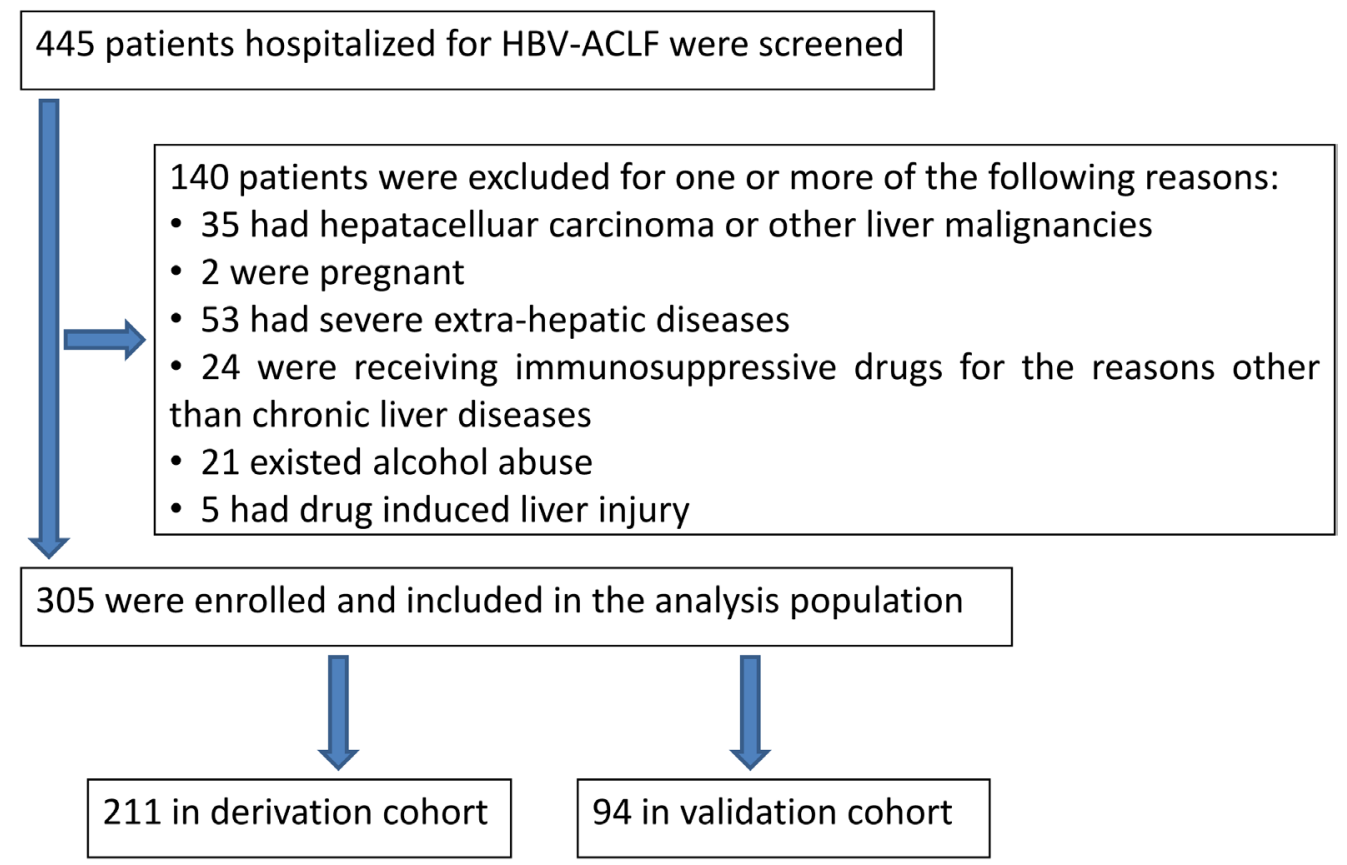

Figure 4: Study flow: diagram showing the process of study selection and exclusion in of HBV-ACLF patients. 
over the first 3 days of hospitalization can reflect the survival rate of ALF [22]. Another study reported that $\triangle \mathrm{MELD}$ is superior to initial MELD and that CTP scores are reliable in patients with advanced cirrhosis [14]. In our study, the initial model was constructed based on the independent predictors of admission mortality. However, the discriminative ability was just moderate (AUROC = 0.7451 ). When $\Delta$ biomarker was calculated and constructed into the model together with baseline risk factors, this model had a great discrimination (AUROC $=0.8475$ ). Taken together, it is suggested that continuous changes in predictive variables can better predict mortality than using static variables.

On admission, the AUROC for MELD, MELD-Na, and CTP scores were $0.6962,0.6862,0.5656$, respectively, which is consistent with the results reported [23]. However, the MELD, MELD-Na and CTP scoring systems are used to predict mortality risk in untreated cirrhotic patients [24-25]. These models did not consider the impact of biomarker changes after diagnosis, HBV-ACLF related complications and in-hospital special treatment on the prognosis. When HBV-ACLF related complications and biomarker ( $\Delta$ biomarker) changes daily within 7 days after diagnosis were established with baseline risk factors according to logistic regression, the HBV-ACLFD model $($ AUROC $=0.8475)$ showed better potential than the established prognostic models like MELD, MELD-Na and CTP scores in a cohort of patients with HBV-ACLF for predicting the 30-day mortality. By the AUROC, these results support that $\Delta$ biomarker and $\mathrm{HE}$ can improve its accuracy in predicting mortality. Thus, the HBV-ACLFD model showed good discrimination in the derivation cohort. When applied to the separate validation cohort of patients with HBV-ACLF, the new model retained good discrimination, accurately distinguishing the ACLF patients who need LT, and grasping the best opportunity for their transplantation.

We also evaluated biochemical and clinical variables via multivariate logistic regression. HE, suspicion of infection, baseline and average daily changes of serum TBiL, INR, serum albumin and blood neutrophils percentage were independent prognostic factors for 30-day mortality. Meanwhile, on multivariate analysis, suspicion of infection is associated with mortality. The hazard ratio for mortality of patients with HBV-ACLF was 30.37 during the first 30-day. It is recommended that patients with high risk of complications and cirrhosis are highly suspected bacterial infection [26].

The systemic inflammatory response syndrome (SIRS) occurs in patients with advanced cirrhosis and is correlated with poor prognosis [27]. The definition of SIRS and sepsis are very difficult because of the following findings [28]: elevated baseline heart rate for hyperdynamic circulatory syndrome; reduced baseline cell count for hypersplenism; hepatic encephalopathy leading to excessive ventilation; cirrhotic patients with mildly elevated body temperature [26]. The suspicion of infection is correlated with a good prognosis [29]. Rapid initiation of appropriate antibiotic therapy is critical to the management of patients suspected of being infected. Delayed and inappropriate treatments are correlated with increased mortality [30].

Moreover, the validation cohort data were collected from three different medical centers of geographic location in China, which means our model is validated in different regions of the population and in different areas of HBV-ACLF lesions. It also improves the accuracy and credibility of our HBV-ACLFD model, which is another principal strength of our model. Because this model is based on retrospective data on a great number of patients with HBV-ACLF from three different medical centers, it also means that homogeneous cohort managed with similar treatment regimens cannot be secured. It is indicated that this model has a broad spectrum of practicality.

However, novel clinical strategies, including artificial liver support system and stem cell transplantation, apply to treat HBV-ACLF now. Our model cannot assess the effect of these novel clinical strategies for accurately predict outcome in patients with HBV-ACLF. Meanwhile, the comparison between the CLIF Consortium ACLF score (CLIF-C ACLFs) and the HBV-ACLFD model should be explored, despite the fact that the CLIF-C ACLFs is helpful to predicting short-term mortality in ACLF patients in Western countries [31-32], where the most common etiology of ACLF is alcoholic liver disease.

In conclusion, we retrospectively deduced and validated the dynamic models of predictive outcomes in patients with HBV-ACLF. This model may be helpful in clinical decision making and risk stratification for patients with HBV-ACLF.

\section{MATERIALS AND METHODS}

All procedures and methods related to this research were accorded morally with current laws as well as the creeds of the Declaration of Helsinki. The research was permitted by the Ethical Committee of Beijing You-An Hospital, Capital Medical University.

\section{Study design and patients selection}

A total of 445 patients who were diagnosed as HBV-ACLF from January 2005 to February 2014 were included in this research. The minimum follow-up period for enrolled patients was 30-day after diagnosis. The diagnosis of cirrhosis was according to a composite of clinical signs and findings provided through laboratory test results, radiologic imaging, endoscopy and liver biopsy.

The entry criteria comprised the following:HBVACLF is defined as ACLF with previously diagnosed or undiagnosed HBV. All enrolled patients met the criteria for ACLF from the consensus recommendations of the Asian 
Pacific Association for the Study of the Liver (APASL) [33]. All treatments were performed based on the criteria of diagnostic and treatment guidelines for ACLF adopted by the Chinese Medical Association [34].

The exclusion criteria were the following: other factors induce severe liver injury, such as alcohol, drugs, hepatoviruses other than HBV, autoimmunity and pregnancy, as well as genetic and metabolic disorders. HBV-ACLF patients with hepatocellular carcinoma, known decompensated cirrhosis prior to onset of acute hepatic insult, age less than 18 years, jaundice induced by hemolytic jaundice and obstructive jaundice, absence of any chronic liver disease on investigations, and prolonged prothrombin time induced by blood system diseases were also excluded. The flowchart for the selection of HBVACLF patients was shown in Figure 4.

Derivation cohort data were screened from Beijing You-an Hospital from January 2005 to January 2013, while validation cohort data was collected from three different medical centers from January 2013 to February 2014. Specifically, 30 cases, 24 cases and 40 cases of HBV-ACLF as validation cohort data were collected from Beijing You-an Hospital, Tianjin Third Central Hospital and the Third Affiliated Hospital of Sun YatSen University, respectively. The derivation cohort was applied to determine the predictors of mortality and thus established a prognostic model.

\section{Observed parameters}

Data on patient demographics, clinical and laboratory variables, evaluation of the underlying chronic liver disease, complications during the medication course, in-hospital special treatment (antiviral therapy, plasma exchange, corticosteroid exposure) were retrospectively collected. All enrolled patients were followed up for a minimum of 30-day by clinic visits or telephone. The outcome (death or survival) of each patient with HBVACLF was documented. Suspicion of infection based on at least one of the following: WBC count $>10,000 / \mathrm{mm} 3$ or $\geq 50 \%$ increase with respect to baseline with a final value $>8,000 / \mathrm{mm} 3$; more than $5 \%$ of band forms; and/or temperature $>37.5^{\circ} \mathrm{C}$.

The results of blood tests performed on the day of diagnosis and within 7 days after diagnosis were recorded. The blood tests consisted of white blood count (WBC), platelet count (PLT), hemoglobin level, blood neutrophils percentage count (NEUT), international normalized ratio (INR), prothrombin time (PT), creatinine level, aspartate transaminase (AST) level, aspartate alanine transaminase (ALT) level, TBil level (total bilirubin), direct bilirubin (DBil) level, cholinesterase, glucose, total cholesterol level, albumin level, triglyceride level, serum sodium level, ammonia level, serum chloride level, serum potassium level, serum magnesium level and total serum calcium level.
Differences of biomarker ( $\Delta$ biomarker) levels within 7 days after diagnosis were calculated. For example, a patient with HBV-ACLF with an ALT of $100 \mathrm{U} / \mathrm{L}$ on the day of admission and $380 \mathrm{U} / \mathrm{L}$ on day 7 after admission, had $\triangle \mathrm{ALT}=(380-100) / 7=40$. In the case of variceal hemorrhage or plasma exchange, the results of blood tests after variceal hemorrhage or plasma exchange were chosen after more than 2 days.

\section{Management protocol}

Patients who were positive for HBV-DNA at presentation underwent antiviral therapy (lamivudine, telbivudine, or entecavir) with informed consent. In order to prevent/treat complications, comprehensive medical interventions were applied, including absolute bed rest, intravenous drop infusion of albumin or plasma, energy supplements, maintenance of acid-base equilibrium or electrolyte, plasma exchange the use of adenosylmethionine, glutathione or branched-chain amino acids to nourish liver cells, as well as antibiotics for infection. Patients with decompensation requiring organ support (such as variceal hemorrhage, HE, hepatorenal syndrome, mechanical ventilationor multiorgan failure) were admitted to ICU.

\section{Calculation of the CTP, MELD and MELD-Na}

The MELD equation was applied to calculate the score of severity: $9.57 \times \ln$ (creatinine, $\mathrm{mg} /$ dl) $+3.78 \times \ln ($ bilirubin, $\mathrm{mg} / \mathrm{dl})+11.2 \times \ln (\mathrm{INR})+6.43$, in which the minimal values were forced to 1.0 for calculation purposes [29]. The MELD-Na equation was constructed based on the $\mathrm{Na}$ and MELD, MELD+1.59 $\times(135-\mathrm{Na})$, with minimum and maximum $\mathrm{Na}$ values as 120 and 135 $\mathrm{mEq} / \mathrm{L}$, respectively [35]. The CTP classification was assessed according to the standard criteria [36].

\section{Procedures}

The derivation cohort was applied to determine the predictors of mortality and thus established a prognostic model. $\Delta$ biomarker was calculated and constructed into the model together with baseline risk factors based on logistic regression, which is our dynamic prognostic model for HBV-ACLF, named the HBV-ACLF dynamic (HBV-ACLFD) model. AUROC were used as a control to compare the predictive values in the HBV-ACLFD model, i.e. MELD score, MELD-Na score and CTP score.

\section{Statistical analysis}

All statistical analyses were conducted by STATA version 13.1. Univariate analyses were applied by appropriate tests to identify the variables which were significantly different in patients who died or survived in the derivation cohort. Multivariable logistic regression 
model was used by taking these predictor variables with the outcome (survived vs. death) through a stepwise forward selection procedure and thereby establish the dynamic prognostic model. The validity of this model was constructed by concordance (c) statistics, which is equivalent to the area under the AUROC curve. C-value $>0.7$ was recommended useful, and the $\mathrm{C}$ - value $>0.8$ would be considered excellent. Then, the performance of this model was finally verified in an independent cohort.

\section{CONFLICTS OF INTEREST}

No potential conflicts (financial, professional, or personal) is relevant to the manuscript.

\section{FUNDING}

The study was supported by the National Natural Science Foundation of China (No. 81470877), the Foundation of Capital Science Committee of China (H020920020890), and Capital Health Research and Development of Special (2011-2018-09).

\section{REFERENCES}

1. Liu Q, Liu Z, Wang T, Wang Q, Shi X, Dao W. Characteristics of acute and sub-acute liver failure in China: nomination, classification and interval. J Gastroenterol Hepatol. 2007;22:2101-6.

2. Olson JC, Kamath PS. Acute-on-chronic liver failure: what are the implications? Curr Gastroenterol Rep. 2012;14:63-6.

3. Sarin SK, Choudhury A. Acute-on-chronic liver failure: terminology, mechanisms and management. Nat Rev Gastroenterol Hepatol. 2016;13:131-49.

4. Bernal W, Jalan R, Quaglia A, Simpson K, Wendon J, Burroughs A. Acute-on-chronic liver failure. Lancet 2015;386:1576-87.

5. Asrani SK, Simonetto DA, Kamath PS. Acute-on-chronic liver failure. Clin Gastroenterol Hepatol. 2015; 13:2128-39.

6. Shalimar, Kumar D, Vadiraja PK, Nayak B, Thakur B, Das P, Datta Gupta S, Panda SK, Acharya SK. Acute on chronic liver failure because of acute hepatic insults: etiologies, course, extrahepatic organ failure and predictors of mortality. J Gastroenterol Hepatol. 2016;31:856-64.

7. Zheng YX, Zhong X, Li YJ, Fan XG. Performance of scoring systems to predict mortality of patients with acute-on-chronic liver failure: a systematic review and meta-analysis. J Gastroenterol Hepatol. 2017. https://doi. org/10.1111/jgh.13786.

8. Wiesner R, Edwards E, Freeman R, Harper A, Kim R, Kamath P, Kremers W, Lake J, Howard T, Merion RM, Wolfe RA, Krom R; United Network for Organ Sharing Liver Disease Severity Score Committee. Model for end-stage liver disease (MELD) and allocation of donor livers. Gastroenterology. 2003;124:91-6.

9. Hong G, Lee KW, Suh S, Yoo T, Kim H, Park MS, Choi Y, Yi NJ, Suh KS. The model for end-stage liver disease scorebased systempredicts short term mortality better than the current Child-Turcotte-Pugh score-based allocation system during waiting for deceased liver transplantation. J Korean Med Sci. 2013;28:1207-12.

10. Marroni $\mathrm{CP}$, de Mello Brandão $\mathrm{AB}$, Hennigen $\mathrm{AW}$, Marroni C, Zanotelli ML, Cantisani G, Fuchs SC; Liver Transplantation Group. MELD scores with incorporation of serum sodium and death prediction in cirrhotic patients on the waiting list for liver transplantation: a single center experience in southern Brazil. Clin Transplant. 2012;26:E395-401.

11. Kim WR, Biggins SW, Kremers WK, Wiesner RH, Kamath PS, Benson JT, Edwards E, Therneau TM. Hyponatremia and mortality among patients on the liver-transplant waiting list. N Engl J Med. 2008;359:1018-26.

12. Hernaez R, Solà E, Moreau R, Ginès P. Acute-on-chronic liver failure: an update. Gut. 2017;66:541-53.

13. Jalan R, Yurdaydin C, Bajaj JS, Ginès P. Toward an improved definition of acute-on-chronic liver failure. Gastroenterology. 2014;147:4-10.

14. Huo TI, Wu JC, Lin HC, Lee FY, Hou MC, Lee PC, Chang FY, Lee SD. Evaluation of the increase in model for endstage liver disease (DeltaMELD) score over time as a prognostic predictor in patients with advanced cirrhosis: risk factor analysis and comparison with initial MELD and Child-Turcotte-Pugh score. J Hepatol. 2005; 42:826-32.

15. Mas A, Rodeg J. Fulminant hepatitis failure. Lancet. 1997; 349:1081-5.

16. Arroyo V, Moreau R, Jalan R, Ginès P; EASL-CLIF Consortium CANONIC Study. Acute-on-chronic liver failure: a new syndrome that will re-classify cirrhosis. J Hepatol. 2015;62:S131-43.

17. Wlodzimirow KA, Eslami S, Abu-Hanna A, Nieuwoudt M, Chamuleau RA. A systematic review on prognostic indicators of acute on chronic liver failure and their predictive value for mortality. Liver Int. 2013;33:40-52.

18. Anand AC, Dhiman RK. Acute on chronic liver failure-what is in a 'definition'?. J Clin Exp Hepatol. 2016;6:233-40.

19. Yamagishi Y, Saito H, Ebinuma H, Kikuchi M, Ojiro K, Kanamori H, Tada S, Horie Y, Kato S, Hibi T. A new prognostic formula for adult acute liver failure using computer tomography-derived hepatic volumetric analysis. J Gastroenterol. 2009;44:615-23.

20. Xia Q, Dai X, Zhang Y, Guo Y, Xu X, Yang Q, Du W, Liu X, Chen Y, Huang J, Li L. A modified MELD model for Chinese pre-ACLF and ACLF patients and it reveals poor prognosis in pre-ACLF patients. PLoS One. 2013;8:e64379.

21. Kumar R, Shalimar, Sharma H, Goyal R, Kumar A, Khanal S, Prakash S, Gupta SD, Panda SK, Acharya SK. Prospective derivation and validation of early dynamic 
model for predicting outcome in patients with acute liver failure. Gut. 2012;61:1068-75.

22. Schiødt FV, Ostapowicz G, Murray N, Satyanarana R, Zaman A, Munoz S, Lee WM. Alpha-fetoprotein and prognosis in acute liver failure. Liver Transpl. 2006;12:1776-81.

23. Xun YH, Shi JP, Li CQ, Li D, Shi WZ, Pan QC, Guo JC, Zang GQ. Prognostic performance of a series of model for end-stage liver disease and respective Delta scores in patients with hepatitis B acute-on-chronic liver failure. Mol Med Rep. 2014;9:1559-68.

24. Zhang QB, Chen YT, Lian GD, Qian CC, Chen SJ, Huang KH. A combination of models for end-stage liver disease and cirrhosis-related complications to predict the prognosis of liver cirrhosis. Clin Res Hepatol Gastroenterol. 2012;36:583-91.

25. Peng Y, Qi X, Tang S, Deng H, Li J, Ning Z, Dai J, Hou F, Zhao J, Wang R, Guo X. Child-Pugh, MELD, and ALBI scores for predicting the in-hospital mortality in cirrhotic patients with acute-on-chronic liver failure. Expert Rev Gastroenterol Hepatol. 2016;10:971-80.

26. Fagiuoli S, Colli A, Bruno R, Burra P, Craxì A, Gaeta GB, Grossi P, Mondelli MU, Puoti M, Sagnelli E, Stefani $\mathrm{S}$, Toniutto P. Management of infections in cirrhotic patients: report of a consensus conference. Dig Liver Dis. 2014;46:204-12.

27. Abdel-Khalek EE, El-Fakhry A, Helaly M, Hamed M, Elbaz O. Systemic inflammatory response syndrome in patients with liver cirrhosis. Arab J Gastroenterol. 2011;12:173-7.

28. Cazzaniga M, Dionigi E, Gobbo G, Fioretti A, Monti V, Salerno F. The systemic inflammatory response syndrome in cirrhotic patients: relationship with their in-hospital outcome. J Hepatol. 2009;51:475-82.

29. Malinchoc M, Kamath PS, Gordon FD, Peine CJ, Rank J, ter Borg PC. A model to predict poor survival in patients undergoing transjugular intrahepatic portosystemic shunts. Hepatology. 2000;31:864-71.
30. European Association for the Study of the Liver. EASL clinical practice guidelines on the management of ascites, spontaneous bacterial peritonitis, and hepatorenal syndrome in cirrhosis. J Hepatol. 2010;53:397-417.

31. Sy E, Ronco JJ, Searle R, Karvellas CJ. Prognostication of critically ill patients with acute-on-chronic liver failure using the Chronic Liver Failure-Sequential Organ Failure Assessment: a Canadian retrospective study. J Crit Care. 2016;36:234-9.

32. Lee M, Lee JH, Oh S, Jang Y, Lee W, Lee HJ, Yoo JJ, Choi WM, Cho YY, Cho Y, Lee DH, Lee YB, Yu SJ, et al. CLIF-SOFA scoring system accurately predicts short-term mortality in acutely decompensated patients with alcoholic cirrhosis: a retrospective analysis. Liver Int. 2015;35:46-57.

33. Sarin SK, Kedarisetty CK, Abbas Z, Amarapurkar D, Bihari C, Chan AC, Chawla YK, Dokmeci AK, Garg H, Ghazinyan H, Hamid S, Kim DJ, Komolmit P. Acute-on-chronic liver failure: consensus recommendations of the Asian Pacific Association for the Study of the Liver (APASL) 2014. Hepatol. Int. 2014;8:453-71.

34. Liver Failure and Artificial Liver Group, Chinese Society of Infectious Diseases, Chinese Medical Association; Severe Liver Diseases and Artificial Liver Group, Chinese Society of Hepatology, Chinese Medical Association. [Diagnostic and treatment guidelines for liver failure (2012 version)]. [Article in Chinese]. Zhonghua Gan Zang Bing Za Zhi. 2013;21:177-83.

35. Biggins SW, Kim WR, Terrault NA, Saab S, Balan V, Schiano T, Benson J, Therneau T, Kremers W, Wiesner R, Kamath P, Klintmalm G. Evidence-based incorporation of serum sodium concentration into MELD. Gastroenterology. 2006;130:1652-60.

36. Pugh RN, Murray-Lyon IM, Dawson JL, Pietroni MC, Williams R. Transection of the oesophagus for bleeding oesophageal varices. Br J Surg. 1973;60:646-9. 